\title{
Complex and Sustained Quantum Beating Patterns in a Classic IVR System: The $\mathbf{3}^{\mathbf{1}} 5^{\mathbf{1}}$ Level in $S_{1} p$-Difluorobenzene.
}

Jonathan Midgley, Julia A. Davies ${ }^{1}$ and Katharine L. Reid

School of Chemistry, University of Nottingham, Nottingham, NG7 2RD, United Kingdom

\begin{abstract}
Using picosecond time-resolved photoelectron imaging we have studied the intramolecular vibrational energy redistribution (IVR) dynamics that occur following the excitation of the $3^{1} 5^{1}$ level which lies $2068 \mathrm{~cm}^{-1}$ above the $S_{1}$ origin in $p$-difluorobenzene. Our technique, which has superior time resolution to that of earlier studies but retains sufficient energy resolution to identify the behavior of individual vibrational states, enables us to determine six distinct beating periods in photoelectron intensity, only one of which has been observed previously. Analysis shows that the IVR dynamics are restricted among only a handful of vibrational levels, despite the relatively high excitation energy. This is deduced to be a consequence of the high symmetry and rigid structure of p-difluorobenzene.
\end{abstract}

\section{TOC graphic:}

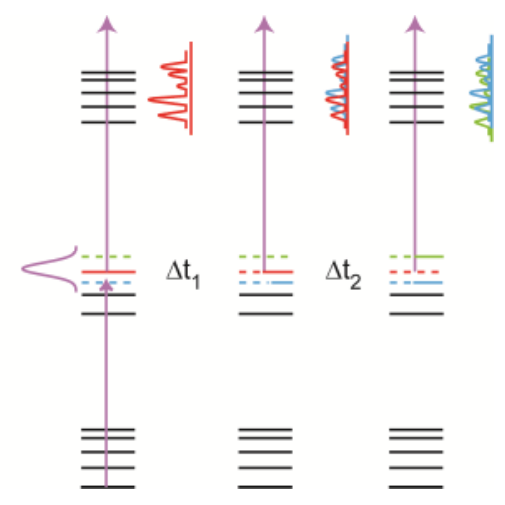

Keywords: Intramolecular, excited state dynamics, photoelectron, ultrafast lasers, timeresolved, vibrational energy

\footnotetext{
${ }^{1}$ Current address: Department of Chemistry, Imperial College London, Exhibition Road, London SW7 2AZ, UK
} 
The dynamics that occur following the photoexcitation of vibrational levels in $\mathrm{S}_{1}$ p-difluorobenzene provide a classic example in the literature on intramolecular vibrational energy redistribution (IVR). ${ }^{1-14}$ Among the levels studied, one found at $2068 \mathrm{~cm}^{-1}$ above the $\mathrm{S}_{1}$ origin has attracted particular interest. ${ }^{10-13}$ This level is usually labelled the $3^{1} 5^{1}$ level, ${ }^{15}$ and involves excitation of one quantum in mode 3 (CF stretching) and one quantum in mode 5 (ring breathing). The dynamics following the excitation of the $3^{1} 5^{1}$ level has been studied in room temperature chemical timing experiments by Parmenter and coworkers, who concluded that it exhibited characteristics of IVR in the regime intermediate between restricted (quantum beating between a few levels) and statistical (exponential decay). ${ }^{7}$ From their experiments an "IVR lifetime" (which they defined as a dephasing time) of 97 ps was deduced. The authors concluded that it should be possible to observe quantum beating superimposed on an exponential decay if the IVR dynamics were studied in a rotationally cold sample. Later work by Knee and coworkers $^{13}$ studied a rotationally cold sample using picosecond time-resolved zero kinetic energy photoelectron (ZEKE) spectroscopy. In this work quantum beats with a period of 105 ps were observed, which decayed slightly over the 800 ps range studied. This behavior is characteristic of two-level restricted IVR on which is superimposed the effects of rotational dephasing. ${ }^{16}$ The Knee study was conducted using laser pulses of $\sim 15$ ps duration and a bandwidth of $\sim 10 \mathrm{~cm}^{-1}$, and experiments were constrained by the wavelength limitations of the laser system. This restricted the ion states that could be monitored, and as a consequence out-of-phase beats were not observed and the dark zero order state causing the oscillations could not be assigned. An obvious candidate for this dark state is $3^{1} 6^{2}$ because the $3^{1} 5^{1}$ and $3^{1} 6^{2}$ states are known to be in Fermi resonance in $S_{1}$. However, the energy separation between the eigenstates resulting from this coupling was determined to be $\sim 6 \mathrm{~cm}^{-1}$ by Knight and Kable; ${ }^{17}$ this is not consistent with the observed quantum beating period of $\sim 105$ ps which corresponds to a separation of $\sim 0.3 \mathrm{~cm}^{-1}$. In this Letter we describe a re-examination of the observed quantum beating pattern, using 1 ps pulses with a bandwidth of $13 \mathrm{~cm}^{-1}$, and report significant additional structure providing further insight into the IVR dynamics.

In Fig. 1 we show a color map of time-dependent photoelectron intensity derived from photoelectron spectra which were measured using a fixed probe wavelength of $255.75 \mathrm{~nm}$ at time delays from 0 to 500 ps in 10 ps steps; see Methods section. This map shows clear 
recurrence structure as a function of time $(t)$, including evidence of additional ion vibrational states that are not observed at $t=0$ growing in at $t>0$. The latter behavior can be seen more clearly in the photoelectron spectra shown in Fig. 2. In these spectra the energy resolution is improved by the use of a longer wavelength in the ionization step. The photoelectron spectrum observed at $t=0$ agrees with that observed in previous work, ${ }^{11}$ and assignments of the prominent ion vibrational states observed at $t=0$ can be made (see Fig. 2). Furthermore, the peaks labelled (i) and (ii), occurring at 1689 and $2132 \mathrm{~cm}^{-1}$ in the $50 \mathrm{ps}$ spectrum, have been assigned to the $28^{2}$ and $6^{1} 28^{2}$ ion states, ${ }^{18}$ where mode 6 is a CCC inplane bend and mode 28 is a $\mathrm{CH}$ out of plane bend.

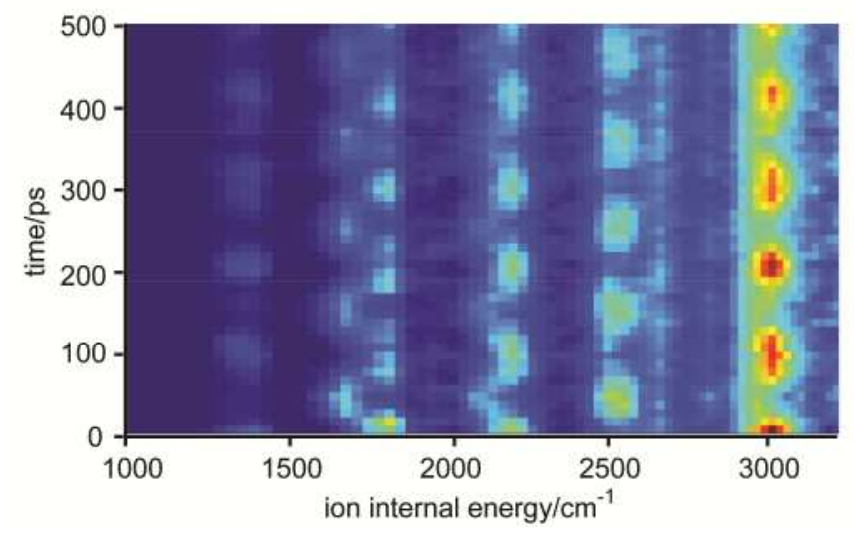

Fig. 1: Color map of time-dependent photoelectron intensity following the excitation of the $3^{1} 5^{1}$ level in $S_{1}$ p-difluorobenzene with a 1 ps pump pulse and ionization with a time delayed 1 ps probe pulse at $255.75 \mathrm{~nm}$.

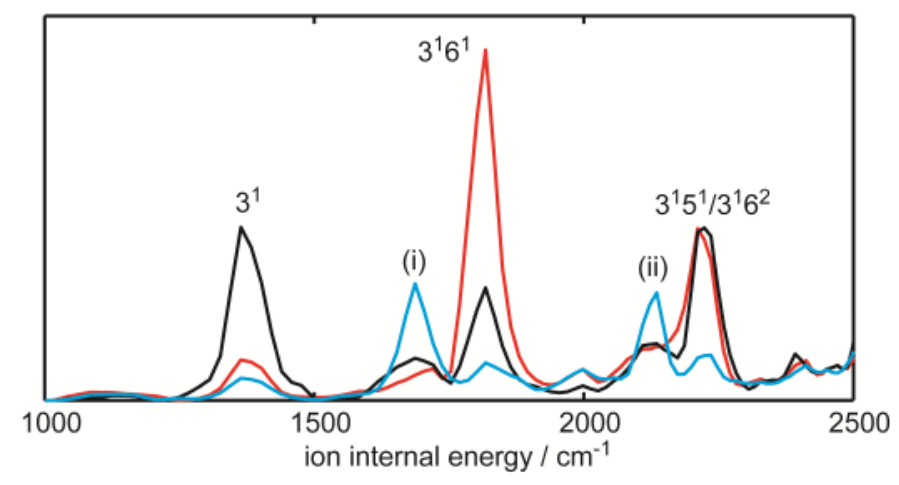

Fig. 2: Photoelectron spectra following the ionization of $S_{1}$ p-difluorobenzene in its $3^{1} 5^{1}$ state using a probe wavelength of $264.7 \mathrm{~nm}$. The probe pulse arrives after the pump pulse by 0 ps (black line), 4 ps (red line) and 50 ps (blue line). 
As discussed in our earlier work, ${ }^{19}$ each ion state is sensitive in different ways to the evolution of the prepared vibrational wavepacket as a consequence of the different Franck Condon factors in operation. This is illustrated in Fig. 3, which shows the intensities of the peaks corresponding to three ion states as a function of time. The time profile shown in Fig. 3 a corresponds to the $3^{1}$ ion state which tracks the evolution of the $S_{1} 3^{1} 5^{1}$ bright state. The time profile shown in Fig. $3 b$ corresponds to the $3^{1} 6^{1}$ ion state which predominantly tracks the evolution of the $S_{1} 3^{1} 6^{2}$ dark state which is not populated at $t=0$. The time profile shown in Fig. $3 c$ corresponds to the $28^{2}$ ion state (peak (i) in Fig. 2, see above), which predominantly tracks the evolution of an $S_{1}$ dark state other than $3^{1} 6^{2}$ whose assignment is discussed below. The traces in Figs. 3a-c show an oscillation with a period of 103 ps, in agreement with earlier work, ${ }^{13}$ on which is superimposed a higher frequency oscillation that is clearly resolved in Figs. 3a and 3b. The high frequency components have opposite phases in Figs. 3a and b, whereas the low frequency components have opposite phases in Figs. 3a and 3c. The trace in Fig. $3 c$ has poorer signal-to-noise, but it is clear that the high frequency oscillation has relatively low intensity in this case.

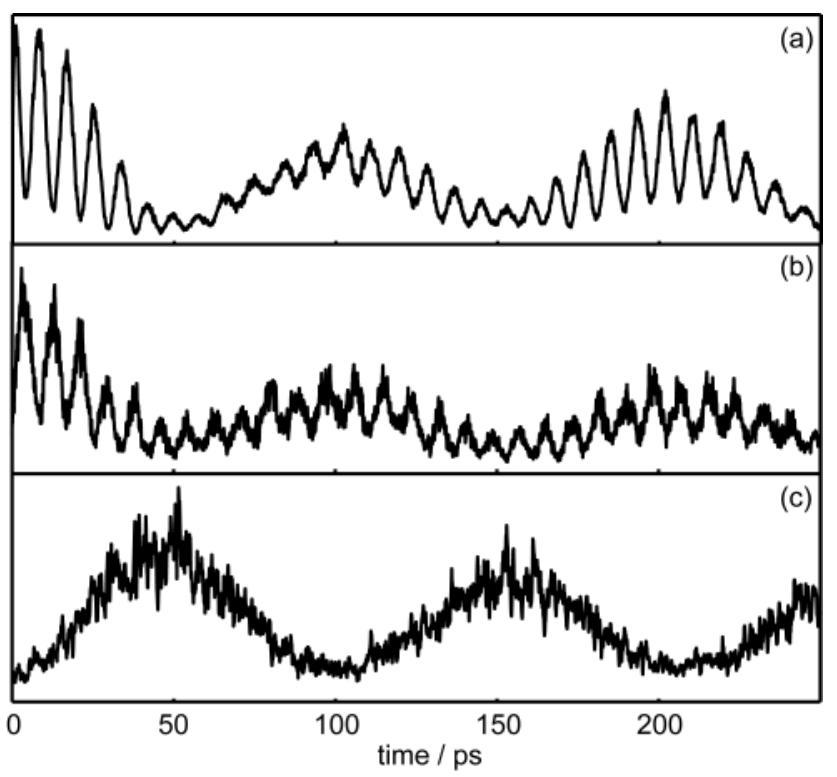

Fig. 3: Time-dependent photoelectron intensity corresponding to: (a) the $3^{1}$ ion peak, (b) the $3^{1} 6^{1}$ ion peak, and (c) the ion peak labelled (i) in Fig. 2 and assigned to $28^{2}$.

The superimposed oscillations that are seen in Fig. 3a can be quantified by means of a Fourier transform (Fig. 4b) which is obtained from the extended time region shown in Fig. 4a. As well as showing a clear peak at $0.33 \mathrm{~cm}^{-1}$ that corresponds to the observed period of 
103 ps, the Fourier transform reveals a peak at $0.16 \mathrm{~cm}^{-1}$ that corresponds to a period of 205 ps, as well as four strong peaks between 3.6 and $4.2 \mathrm{~cm}^{-1}$ corresponding to periods of $8.5,8.1,9.2$, and 8.8 ps (in order of decreasing relative contribution), meaning that overall six clear oscillation periods are observed. This is an immediate indication that the prepared wavepacket contains multiple levels, and not just the two known components of the Fermi resonance. Although minor peaks are also observed at 0.44 and $4.3 \mathrm{~cm}^{-1}$ in Fig. $4 \mathrm{~b}$, they are not observed in Fourier transforms of the time profiles for any of the other ion states. These peaks may indicate very weakly coupled states, or may be artefacts resulting from the Fourier transform procedure. It is clear from the Fourier transform that there is no component close to $6 \mathrm{~cm}^{-1}$ which is the previously determined eigenstate energy separation for the $3^{1} 5^{1} / 3^{1} 6^{2}$ Fermi resonance. ${ }^{17}$ In Fig. $4 a$, the behavior of the beating pattern over an extended time indicates that the population remains within a small subset of levels, and does not leak out into a bath of states. This is characteristic of a restricted IVR process and is contrary to the expectation for intermediate case IVR. The slight decay in the beat amplitude was also observed by Knee and coworkers ${ }^{13}$ and is a consequence of rotational dephasing. ${ }^{16}$
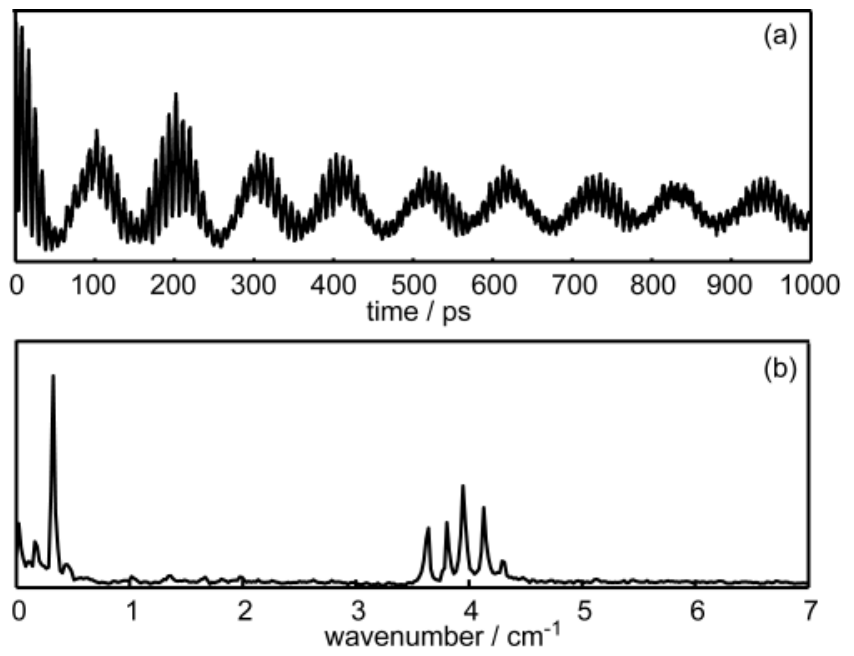

Fig. 4: (a) The time dependent intensity of the photoelectron peak corresponding to the $3^{1}$ ion state over an extended time range, and (b) its Fourier transform.

The $28^{2}$ ion state is not expected to have significant Franck Condon overlap with either the $3^{1} 5^{1}$ or the $3^{1} 6^{2}$ state in $S_{1}$, and so its time behavior (Fig. 3c) must result from tracking a second dark state in $\mathrm{S}_{1}$. The most favorable Franck Condon overlap is expected to be with the $28^{2}$ state in $S_{1}$, but the $S_{1}$ frequency of mode 28 has been determined to be $619 \mathrm{~cm}^{-1}$ by 
Knight and Kable, ${ }^{17}$ which would put the $28^{2}$ state well outside the $\sim 13 \mathrm{~cm}^{-1}$ bandwidth of the pump laser. The level $5^{1} 28^{2}$, which is expected to appear at $\sim 2056 \mathrm{~cm}^{-1}$ in $\mathrm{S}_{1}$, is however expected to lie within the bandwidth, is a plausible candidate for coupling to the $3^{1} 5^{1}$ bright state, and is expected to give rise to population of the $28^{2}$ ion state. The dominance of the 103 ps oscillation in Fig. $3 \mathrm{c}$ is consistent with the $5^{1} 28$ state being closest in energy to the prepared $3^{1} 5^{1}$ bright state, to which it must be strongly coupled. Similar observations have been made in our earlier work. ${ }^{19}$ Because of the known coupling between $5^{1}$ and $6^{2}$ in $\mathrm{S}_{1}$ the presence of $5^{1} 28^{2}$ implies the presence of $6^{2} 28^{2}$ as well, although the latter state is predicted to have a much smaller Franck Condon overlap with the $28^{2}$ ion state. ${ }^{18}$

Figure 5 shows an energy level diagram for the four states involved in the IVR process. For such a scheme, the six energy separations between the four eigenstates: $\Delta E_{12}, \Delta E_{13}, \Delta E_{14}$, $\Delta E_{23}, \Delta E_{24}, \Delta E_{34}$, are related to the the observed oscillation periods, $\tau_{n m}$, by $\tau_{n m}=h / \Delta E_{n m}$. The deduced values of $\tau_{\mathrm{nm}}$ and $\Delta E_{n m}$ together with suggested assignments are listed in Table 1. This Table also contains the relative contributions of each component which have been determined from a fit to the data shown in Fig. 4a. The eigenstate energy separation resulting from the coupling of the $3^{1} 5^{1}$ and $3^{1} 6^{2}$ states is accurately determined in this work to be $3.95 \pm 0.3 \mathrm{~cm}^{-1}$. Furthermore, the dark state associated with the 103 ps quantum beats which was unassigned by Knee and coworkers ${ }^{13}$ can now be identified as $5^{1} 28^{2}$.

We thus conclude that the $3^{1} 5^{1}$ state in $S_{1}$ p-difluorobenzene is strongly coupled to three other vibrational states $\left(3^{1} 6^{2}, 5^{1} 28^{2}\right.$ and $\left.6^{2} 28^{2}\right)$ and that it undergoes restricted IVR. This is surprising given the high excitation energy $\left(2068 \mathrm{~cm}^{-1}\right)$ which is well above the statistical IVR threshold for many other small polyatomic molecules. ${ }^{20}$ Analogous observations were made in anthracene, ${ }^{21}$ leading us to infer that the rigid structure and high symmetry of $\mathrm{p}$-difluorobenzene is likely to be the determining factor in this behavior. 


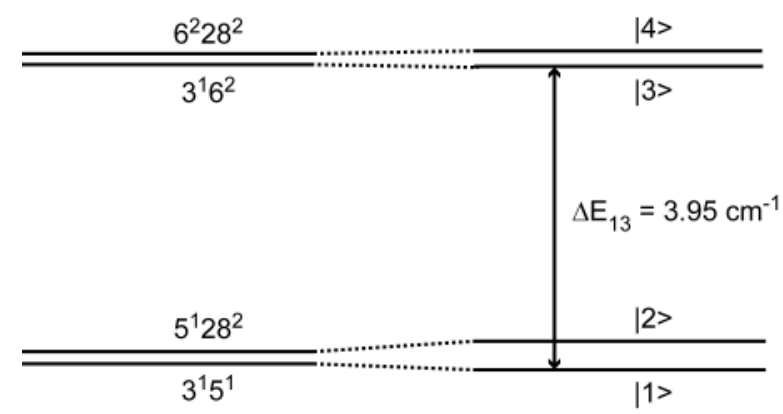

Fig. 5: An energy level diagram for the four states involved in the IVR process. The dotted lines serve to indicate the zero order state (left) that has the largest contribution to each eigenstate (right).

Table 1: Oscillation periods and eigenstate energy separations deduced from the Fourier transform shown in Fig. 4. Likely assignments in terms of the dominant zero-order states are also given.

\begin{tabular}{|c|c|c|c|c|}
\hline $\begin{array}{c}\tau_{\mathrm{nm}} \\
/ \mathrm{ps}\end{array}$ & $\begin{array}{c}\Delta \mathrm{E}_{\mathrm{nm}} \\
/ \mathrm{cm}^{-1}\end{array}$ & $\begin{array}{c}\text { Relative } \\
\text { contribution }\end{array}$ & $\begin{array}{c}\text { Eigenstates } \\
(\mathrm{n}, \mathrm{m})\end{array}$ & $\begin{array}{c}\text { Zero-order } \\
\text { states }\end{array}$ \\
\hline 103 & 0.33 & $\equiv 1.0$ & $(1,2)$ & $3^{1} 5^{1}, 5^{1} 28^{2}$ \\
\hline 205 & 0.16 & 0.24 & $(3,4)$ & $3^{1} 6^{2}, 6^{2} 28^{2}$ \\
\hline 9.2 & 3.64 & 0.37 & $(2,3)$ & $5^{1} 28^{2}, 3^{1} 6^{2}$ \\
\hline 8.8 & 3.81 & 0.26 & $(2,4)$ & $5^{1} 28^{2}, 6^{2} 28^{2}$ \\
\hline 8.5 & 3.95 & 0.71 & $(1,3)$ & $3^{1} 5^{1}, 3^{1} 6^{2}$ \\
\hline 8.1 & 4.13 & 0.48 & $(1,4)$ & $3^{1} 5^{1}, 6^{2} 28^{2}$ \\
\hline
\end{tabular}

\section{Experimental method}

Our experimental procedure has been described elsewhere. ${ }^{19}$ Briefly, a picosecond Ti:Sapph laser system (Coherent) pumps two optical parametric amplifier systems (Light Conversion) which output independently tunable UV pump and probe beams. The measured laser 
bandwidth is $\sim 13 \mathrm{~cm}^{-1}$ and the pulse duration is $1 \mathrm{ps}$. In this work, a pump wavelength of $257.0 \mathrm{~nm}$ is used to prepare $\mathrm{p}$-difluorobenzene in the $3^{1} 5^{1}$ level in $S_{1}$, whilst the probe beam is used to ionize the excited molecules using a wavelength in the range 255 to $275 \mathrm{~nm}$. A motorised delay stage (Standa) achieves a variable time delay ( -20 to $+1000 \mathrm{ps})$ between the pump and probe laser pulses. The vertically-polarised, co-propagating laser beams are focussed into a photoelectron spectrometer where they are spatially and temporally overlapped with a pulsed molecular beam of p-difluorobenzene seeded in He. Photoelectron images are measured using a velocity map imaging photoelectron spectrometer, which has been described in detail in previous work. ${ }^{22}$ Time-resolved photoelectron spectra and angular distributions are extracted from the measured images; the latter have been discussed elsewhere. ${ }^{12}$ In addition, delay stage scans, in which only those photoelectrons striking the central part of the detector are monitored, provide timedependent photoelectron intensity corresponding to the formation of a selected ion vibrational state; this is achieved by careful choice of the probe wavelength.

\section{Acknowledgements}

This work was supported by EPSRC grant EP/E046150.

\section{References}

1. Coveleskie, R. A.; Dolson, D. A.; Parmenter, C. S., A Direct View of Intramolecular Vibrational Redistribution in $\mathrm{S}_{1}$ Para-Difluorobenzene. J. Chem. Phys. 1980, 72, 5774-5775. 2. Coveleskie, R. A.; Dolson, D. A.; Parmenter, C. S., Chem. Timing .2. The Picosecond Dynamics of Intramolecular Vibrational Redistribution in $\mathrm{S}_{1}$ Para-Difluorobenzene. J. Phys. Chem. 1985, 89, 655-665.

3. Coveleskie, R. A.; Parmenter, C. S., Single Vibronic Level Fluorescence Spectra from the $S_{1}\left({ }^{1} B_{2 u}\right)$ State Of Para-Difluorobenzene Vapor. J. Molec. Spec. 1981, 86, 86-114.

4. Davidson, E. R.; Elston, H. J.; Parmenter, C. S., Harmonic Mode Scrambling in ParaDifluorobenzene. Chem. Phys. Lett. 1992, 197, 123-130.

5. Dolson, D. A.; Holtzclaw, K. W.; Moss, D. B.; Parmenter, C. S., Chem. Timing .4. The Rovibronic Level Structure associated with Intramolecular Vibrational Redistribution in S1Para-Difluorobenzene. J. Chem. Phys. 1986, 84, 1119-1132. 
6. Holtzclaw, K. W.; Parmenter, C. S., A Time-Resolved Fluorescence Search for Intramolecular Vibrational Redistribution in $\mathrm{S}_{1}$ Para-Difluorobenzene Vapor. J. Phys. Chem. $1984,88,3182-3185$.

7. Holtzclaw, K. W.; Parmenter, C. S., Chem. Timing .3. The Picosecond Dynamics of Intramolecular Vibrational Redistribution from 11 Levels in $S_{1}$ Para-Difluorobenzene Vapor. J. Chem. Phys. 1986, 84, 1099-1118.

8. Fujii, M.; Ebata, T.; Mikami, N.; Ito, M.; Kable, S. H.; Lawrance, W. D.; Parsons, T. B.; Knight, A. E. W., Mode-Dependent Intramolecular Vibrational Redistribution in the $\mathrm{S}_{1}$ State of Jet-Cooled Para-Difluorobenzene. J. Phys. Chem. 1984, 88, 2937-2940.

9. Kable, S. H.; Lawrance, W. D.; Knight, A. E. W., Evidence for Mode-Specific Intramolecular Vibrational Redistribution in $S_{1}$ p-Difluorobenzene. J. Phys. Chem. 1982, 86, 1244-1247.

10. Nathanson, G. M.; McClelland, G. M., The Role of Rotational Motion in Intramolecular Energy-Transfer - Measurements of Fluorescence Polarization from pDifluorobenzene. Chem. Phys. Lett. 1985, 114, 441-445.

11. Reid, K. L.; Field, T. A.; Towrie, M.; Matousek, P., Photoelectron Angular Distributions as a Probe of Alignment Evolution in a Polyatomic Molecule: Picosecond Time- and AngleResolved Photoelectron Spectroscopy of $S_{1}$ Para-Difluorobenzene. J. Chem. Phys. 1999, 111, 1438-1445.

12. Midgley, J.; Davies, J. A.; Reid, K. L., Comment On "Photoelectron Angular Distributions as a Probe of Alignment in a Polyatomic Molecule: Picosecond Time- and Angle-Resolved Photoelectron Spectroscopy of $S_{1}$ p-Difluorobenzene" J. Chem. Phys. 111, 1438 (1999). J. Chem. Phys. 2013, 139, 117101.

13. Zhang, X.; Smith, J. M.; Knee, J. L., Picosecond Vibrational Dynamics of Several $S_{1}$ Bands in Jet-Cooled p-Difluorobenzene. J. Chem. Phys. 1994, 100, 2429-2436.

14. Baskin, J. S.; Rose, T. S.; Zewail, A. H., Picosecond IVR Dynamics of paraDifluorobenzene and para-Fluorotoluene in a Molecular-Beam - Comparison with Chem. Timing Data. J. Chem. Phys. 1988, 88, 1458-1459.

15. Mulliken, R. S., Report on Notation for the Spectra of Polyatomic Molecules. J. Chem. Phys. 1955, 23, 1997-2011.

16. Felker, P. M.; Zewail, A. H., Dynamics of Intramolecular Vibrational Energy Redistribution (IVR) .3. Role of Molecular Rotations. J. Chem. Phys. 1985, 82, 2994-3002. 
17. Knight, A. E. W.; Kable, S. H., The $S_{1}-S_{0}\left({ }^{1} B_{2 u}-{ }^{1} A_{g}\right)$ Transition of para-Difluorobenzene Cooled in a Supersonic Free Jet Expansion - Excitation And Dispersed Fluorescence-Spectra, Vibrational Assignments, Fermi Resonances, and Forbidden Transitions. J. Chem. Phys. 1988, $89,7139-7160$.

18. Midgley, J. PhD, University of Nottingham, 2014.

19. Davies, J. A.; Green, A. M.; Reid, K. L., Deducing Anharmonic Coupling Matrix Elements from Picosecond Time-Resolved Photoelectron Spectra. Phys. Chem. Chem. Phys. 2010, 12, 9872.

20. Keske, J. C.; Pate, B. H., Decoding the Dynamical Information Embedded in Highly Mixed Quantum States. Annu. Rev. Phys. Chem. 2000, 51, 323-353.

21. Lambert, W. R.; Felker, P. M.; Zewail, A. H., Picosecond Excitation and Selective Intramolecular Rates in Supersonic Molecular-Beams .1. SVL Fluorescence Spectra and Lifetimes of Anthracene and Deuterated Anthracenes. J. Chem. Phys. 1984, 81, 2209-2216. 22. Bellm, S. M.; Reid, K. L., Evaluation of the Use of Photoelectron Imaging in Obtaining Photoelectron Spectra and Angular Distributions: Comparison with the Field-Free Time-OfFlight Method. Chem. Phys. Lett. 2004, 395, 253-258. 